\title{
High-Speed Rolling Bearing Test Rigs with Contactless Signal Transmission for Measuring the Inner Ring Temperature
}

\author{
Prof. Dr.-Ing. C. Brecher ${ }^{1}$, Dipl.-Ing. M. Fey ${ }^{1}$, M.Sc. A. Hassis ${ }^{1}$, Dr.-Ing. S. Bonerz ${ }^{2}$ \\ ${ }^{1}$ Werkzeugmaschinenlabor WZL der RWTH Aachen, Steinbachstraße 19, 52074 Aachen, Germany \\ ${ }^{2}$ OTT-JAKOB Spanntechnik GmbH, Industriestraße 3-7, 87663 Lengenwang, Germany \\ Corresponding contact: a.hassis@wzl.rwth-aachen.de
}

\begin{abstract}
:
Main spindles and their bearings are of vital importance for the performance of machine tools and machining centers. Thus increasing demands on the machines' productivity and reliability directly affect the required bearing properties. The development and testing of rolling bearings in the speed range up to $n \times d_{\mathrm{m}}=2,7 \cdot 10^{6} \mathrm{~mm} / \mathrm{min}$ requires highly specialized test rigs to investigate the bearings' operating behavior. In such test rigs a customized telemetry system is used to continuously measure the test bearing's inner ring temperature. The system uses a shaft integrated bridge circuit with a Pt1000-temperature sensor. The temperature signal is made available as an analog and digital output. This system allows for a deeper understanding of the inner ring excess temperature which is of vital importance for the speed rating of high speed rolling bearings especially of such with small contact angles.
\end{abstract}

Key words: high speed application, test rig, temperature measurement, rolling bearing

\section{Introduction}

The urge for higher productivity in the manufacturing industry leads to higher demands on machine tools and machining centers used there. As core components of these machines, main spindles and thus their bearings are directly affect by these developments. Two ways to increase the productivity are to increase the material removal rate or to decrease service and failure related machine downtimes. The first strategy is realized with high speed cutting (HSC) machining. The latter requires an increase of the components' reliability.

In typical HSC applications a main spindle with the very common bearing seat diameter of $70 \mathrm{~mm}$ (suitable e.g. for a HSK 63 tool interface) rotates at speeds up $30,000 \mathrm{rpm}$ ( $\triangleq n \times d_{\mathrm{m}}=2,7 \cdot 10^{6} \mathrm{~mm} / \mathrm{min}$ for $d_{\mathrm{m}}=90 \mathrm{~mm}$ ).

Friction induced heat is one of the main factors limiting the speed rating of high speed rolling bearings. Besides the fact that excessive bearing temperatures may lead to the deformation or destruction of bearing components, the bearing induced heat also affects the operating behavior of the spindle and the machine. Additionally, bearings with a small contact angle, which is zero for cylindrical roller bearings, tend to seize if the thermally induced radial growth of the inner ring largely excesses that of the outer ring.

The experimental investigation of such bearings requires the usage of specialized test rigs. These test rigs must be mechanically suitable for high rotational speeds and must ensure a continiously good quality of the measuring signals at all speeds.

\section{Main spindles and spindle bearings}

Main spindles for HSC applications are most commonly designed as motor spindles. In conventional spindles the drive motor and the spindle itself are two different components connected by a coupling or a gear or belt transmission. In motor spindles the drive motor is an integral part of the spindle. Not only is this a main requirement for highest speed ratings. As Fig. 1 shows it also allows for a compact cartridge like design that is a prerequisite for a simplified and compact machine design.

One main factor affecting the operating behavior of main spindles is temperature. From Fig. 1 it becomes obvious that there are three main sources of heat generation or heat input in the spindle. First, heat from the cutting process is transferred to the spindle shaft via the tool and the tool interface. Secondly, the 
mechanical friction inside the bearings causes a heat up of the bearings and its surroundings. Third the integrated drive motor produces heat due to the electrical resistance in the rotor and the stator and the magnetic losses.

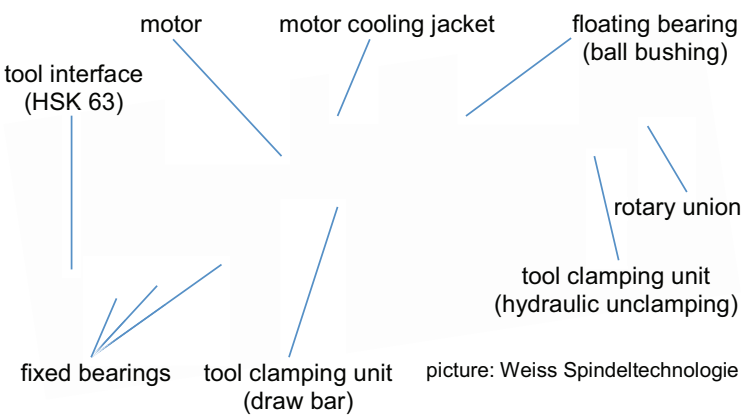

Fig. 1. Main components of a main spindle

Generally, the stator of the motor and in some cases the outer rings of the bearings are fluid cooled. In a very limited number of applications there is also an active cooling of the shaft but in general heat can only be transferred from the shaft by convection and heat dissipation via the bearings. Thus the shaft and thereby the inner rings of the bearings will usually operate at higher temperatures than the housing and the bearings' outer rings.

There are two types of bearings established for the use in high speed spindles. The first and most common type are angular contact ball bearings with a contact angle of $15^{\circ}$ to $25^{\circ}$.

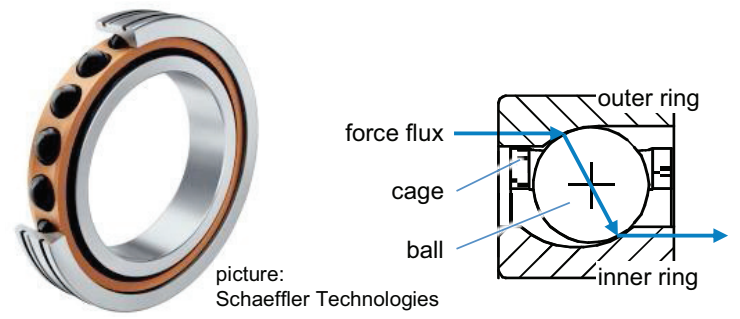

Fig. 2. General spindle bearing geometry

As depicted in Fig. 2 these so called spindle bearings can carry axial and radial loads. Since they can only carry axial loads in one direction they have to be used as preloaded pairs. With a larger radial expansion of the inner ring compared to the outer ring, induced by an excess temperature or centrifugal forces, the contact point between the ball and the outer ring shifts to the apex of the outer ring groove. At the same time the clearance in the bearing decreases. Thus at higher inner ring excess temperatures the clearance can completely be used up. A further radial growth of the inner ring will then lead to a negative clearance what results in increased contact pressures between the balls and the rings. On the one hand, this can lead to fatigue damages. On the other hand, the increased load will lead to increased frictional losses and thereby worsen the temperature imbalance.

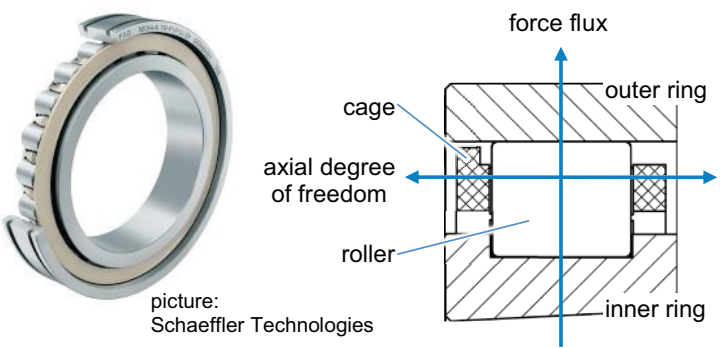

Fig. 3. Cylindrical roller bearing for high speed applications

This problem becomes more critical the smaller the contact angle is. Whereas it can sometimes occur in $15^{\circ}$ spindle bearings it is crucial in cylindrical roller bearings that have a contact angle of $0^{\circ}$. It can be seen from Fig. 3 that any amount of radial expansion of the inner ring that excels that of the outer ring will lead to a reduction of bearing clearance. To achieve proper radial stiffness, many cylindrical roller bearings in main spindles are mounted with an radial preload. Thus they already feature a negative clearance at idle conditions and any inner ring excess temperature leads to an increase in bearing load.

Due to the excess temperature the shaft of a motor spindle will undergo a larger thermal growth in axial and radial direction as the housing. In a setup as depicted in Fig. 1 with an arrangement of preloaded spindle bearings this behavior will considerably affect the bearings' preload. Hence the differences in axial thermal expansion have to be compensated by an axially floating rear bearing. In a setup with only spindle bearings this can be realized by a ball bushing housing the rear bearing's outer ring. With preload springs the axial bearing preload can be kept relatively stable over a wide range of axial expansion. Though the heat dissipation from the rear bearing's outer ring to the housing is very limited due to the air gap between the bushing and the housing. This can contribute to thermal problems with the floating bearings of such spindles.

In contrast, cylindrical roller bearings allow for an axial movement of the inner ring relative to the outer ring without any additional components. Not only does this simplify the spindle design it also enables a better heat transfer from the outer ring to the bearing. Thus despite possible problems resulting from inner ring excess temperatures, cylindrical roller bearings are relatively common as floating bearings in motor spindles for medium speeds. 


\section{Test rigs for high speed rolling bearings}

In the past, one main goal of the development of spindle bearings and high speed cylindrical roller bearings has been the increase of the speed rating. Starting from spindle bearings with large steel balls the reduction of the ball size and a shift in ball material from steel (e.g. $100 \mathrm{Cr} 6$ ) to ceramics (e.g. $\mathrm{Si}_{3} \mathrm{~N}_{4}$ ) led to a considerable reduction of the ball mass and an improvement of the tribological conditions inside the bearings. Combined with optimized raceway geometries this enabled an increase in speed rating by about $75 \%$ (FAG B $7014 \mathrm{E}$ compared to FAG HC 7014 E) [1].

Besides the obvious shift from steel rollers to ceramic rollers, increasing the speed rating of cylindrical roller bearings is very difficult. Besides measures inside the spindle like active cooled shafts, decreasing the radial stiffness of the bearing is a very promising way. It will not decrease the inner ring excess temperature but it will make the bearing more tolerant towards inner ring excess temperatures [2].

To investigate the bearings' speed-temperature behavior in the design or process or for comparing different bearings, test runs on test benches are essential. The FE 8 and FE 9 test benches standardized in DIN 51819-1 [3] and DIN 51821-1 [4] feature several sensors for outer ring temperature and bearing friction moment. They are used to determine the bearing wear or the suitability of lubricants for given operating conditions. However, due to their mechanical design they are neither suitable for high speed tests nor do they allow for a measurement of the bearings' inner ring temperatures.

The development and testing of high speed rolling bearings has been in the focus of WZL for many years. Thus several designs for appropriate high speed test rigs have been developed over the years.

For the investigation of spindle bearings with optimized internal geometries, Rossaint [5] uses the test rig depicted in Fig. 4. At shaft speeds up to $35,000 \mathrm{rpm}$ it allows for the measurement of the outer and inner ring temperature of the test bearing. By means of a hydrostatic bearing housing the test bearing's outer ring in combination with a strain gauge on a bending beam it becomes possible to measure the friction moment of the single test bearing. This is a major difference compared to other rolling bearing test rigs where the friction moment can only be measured as the sum moment of two or more bearings or where the friction moment of several bearings has to be derived from the drive power and speed [3], [4], [6].

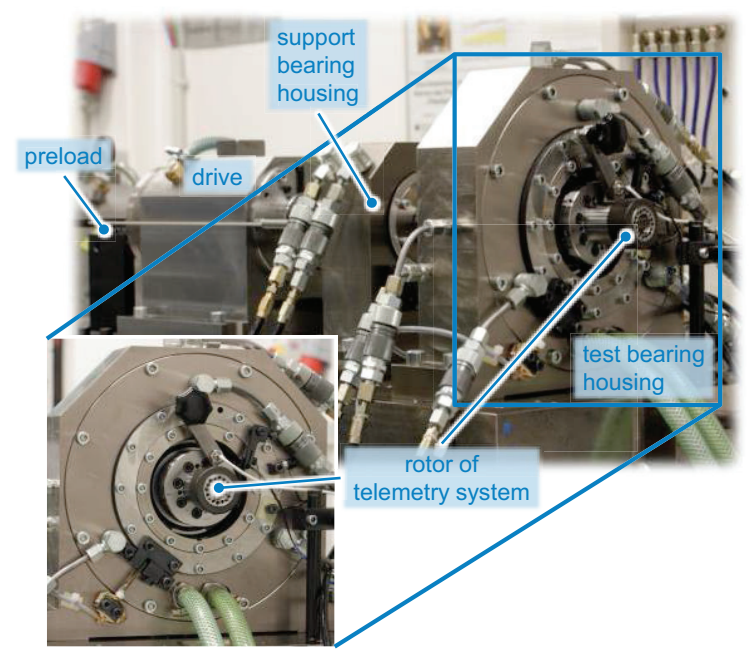

Fig. 4. Test rig for high speed bearings

Another ongoing project deals with the service life of grease lubricated hybrid spindle bearings (i. e. bearings with steel rings and ceramic balls) [7], [8]. Although such bearings are widely used in main spindles their speed rating is considerably lower than that of oil-air-lubricated hybrid bearings. The aim of the project is to investigate possible relations between the grease formulation, the tribological conditions inside the bearing and the operating behavior of the bearing.

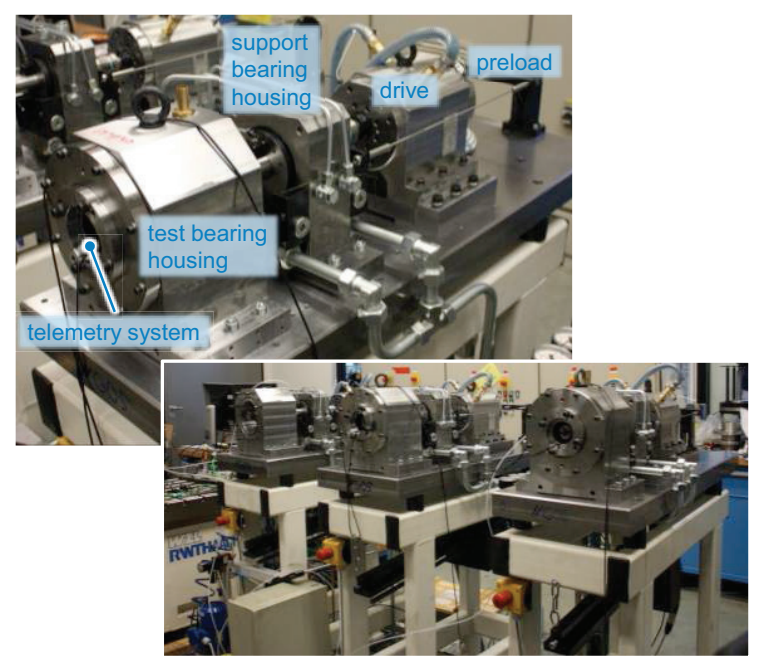

Fig. 5. Multiple test rigs for endurance testing of high speed rolling bearings

One key figure to describe a bearing's operating behavior is the grease service life. To determine the grease service life, bearings are operated at given conditions until the failure of the grease to proper lubricate the rolling contacts leads to a rapid growth of friction and thereby a rapid growth of bearing temperature. Due to the statistical character of the grease service life following a Weibull distribution it is 
recommended to run at least five tests for each combination of grease and operating conditions [9]. To allow for the efficient testing of a larger number of bearings, three test rigs of the same design were built for this project.

As depicted in Fig. 5 these test rigs follow the same design pattern as the one shown in Fig. 4. The main difference is that they do not feature the possibility to measure the bearings friction moment due to the complexity of the setup required.

Both test rig designs feature separate housings for the test and support bearing what reduces thermal interdependencies to a minimum. The floatability of the support bearing is realized by a hydrostatic radial bearing housing the support bearing's outer ring. Even with springs with a low stiffness the preload in spring preloaded bearing arrangements is not ideally constant at different temperature levels. Thus these test rigs feature a preload system of steel cables and a mass that can be moved on a lever. With this gravity based solution the preload can be kept constant for any axial shaft expansion. Additionally it allows for preload alternations during the test run.

\section{Telemetry system for measuring the inner ring temperature}

One key component of the above mentioned test rigs is a telemetry system to measure the test bearings inner ring temperature. Whereas measuring the outer ring temperature can simply be realized by placing a temperature sensor that directly contacts the outer ring in a bore in the housing, the high rotating speeds of the shaft and the inner ring make special demands on the inner ring temperature measurement system.

The difficulty does not result from measuring the temperature but from transferring the signal from the rotating shaft to the static environment and the data acquisition hardware. Common sliding contacts using copper or carbon brushes can not be applied here. The combination of very high circumferential speeds (e.g. $30,000 \mathrm{rpm}$ at $30 \mathrm{~mm}$ shaft diameter) and long running test (typically $>1,000 \mathrm{~h}$ ) would lead to extreme wear rates and far too short service lives. In addition to that, the test rigs operate in an environment where an oil or grease contamination of the contacts can not be precluded.

Telemetry systems also have to fulfill certain requirements to be suitable for the given application: Due to the high rotating speeds they must allow for a radial growth of the rotor. Combined with possible misalignments of the shaft and the thermal and load induced shift of the shaft this requires an appropriate air gap between the rotor and the stator of the system. Additionally, the endurance test rigs are designed to feature the option for internal shaft cooling what makes a center bore in the shafts necessary. The rotor of the telemetry system may not block this bore.

In a cooperation between OTT-JAKOB Spanntechnik and WZL a telemetry system was modified for temperature measurements [10]. The system was originally deigned to measure the drawing force in gas spring equipped draw bar systems for main spindle tool clamping systems.

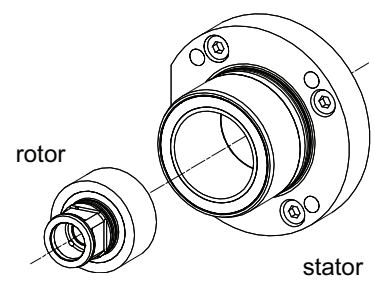

Fig. 6. Mechanical design of the telemetry system

As depicted in Fig. 6 the system consists of a stator with a center bore that is bolted to the test rig housing and a rotor that is mounted on the shaft.

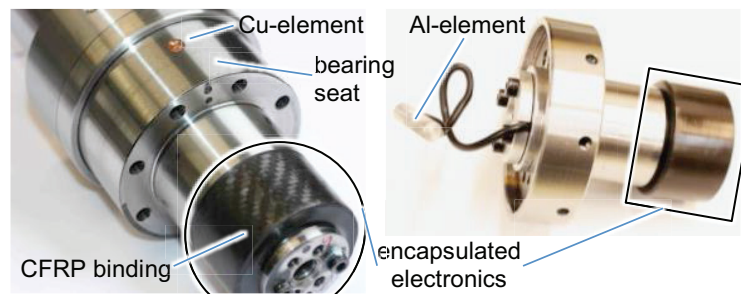

Fig. 7. Shaft integrated telemetry rotor (left), multi application telemetry rotor (right)

Fig. 7 shows the different ways of shaft integration of the rotor that were realized for different applications: In the shaft integrated solution, the telemetry rotor is bolted to the shaft and all the wires running from the rotor to the actual temperature sensor that is located at the rear end of the Cu-element are completely encapsulated. This setup allows for a very good balancing of the shaft. In the multi-application design, the telemetry rotor can be bolted to different shafts what makes the system more flexible if different shafts are to be used on the same test rig.

In both cases it is not possible to directly measure the surface temperature of the inner ring inner surface. An element made of material with good thermal conductivity is the part being in direct contact with the inner ring. The Pt1000temperature sensor is glued to this element. As it will be shown in the following section, this 
does not cause any major measuring errors. In case of the spindle integrated system, the Cuelement is mounted flush with the surface of the bearing seat. In case of the multi application rotor, the Al-element carrying the temperature sensor is pushed to the inner ring bore surface by centrifugal forces.

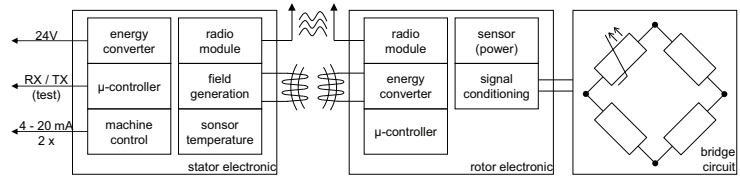

Fig. 8. Electrical setup of the telemetry system

In its original setup the bridge circuit of the rotor shown in Fig. 8 consisted of four strain gauges for strain measurements to determine forces. For the application described here, three of this strain gauges were replaced with constant $1 \mathrm{k} \Omega$-resistances. The fourth was replaced by a Pt1000-temperature sensor.

Via an electric field of the stator a voltage is induced in the rotor. This voltage is used to operate the radio module and as supply voltage for the bridge circuit. The bridge voltage of the bridge circuit is digitalized and transferred to the stator by radio. The stator itself features several signal outputs: First, the digital signal can directly be used. Secondly, the stator integrated D/A converter gives a 4...20 mA output current that is directly proportional to the digital output signal. In the given application this current output is further converted to a voltage signal that is acquired by the test rig's control hardware. For evaluating the results, this voltage $U_{\text {meas }}$ is numerically transferred to the inner ring temperature $T_{\mathrm{IR}}$ via the output current $I$, the digital signal $S$, the measured bridge voltage inside the rotor $U_{\mathrm{br}}$, and the resistance $R_{\mathrm{Pt} 1000}$ of the Pt1000-element. Thus the chain of signal evaluation writes as:

$U_{\text {meas }} \rightarrow I \rightarrow S \rightarrow U_{\mathrm{br}} \rightarrow R_{\mathrm{Pt} 1000} \rightarrow T_{\mathrm{IR}}$

One key feature of the system is the special antenna design with an almost angle independent antenna structure. This ensures a constant signal strength independently of the angular position of the rotor in the stator. This a prerequisite for good signal quality at high speeds. In the current design, the speed rating of the system is only limited by the mechanical strength of the components used. As shown in Fig. 7 (left) the strength of the rotor is ensured by a CFRP binding housing the encapsulated rotor electronics.

\section{Exemplary test results}

The indirect temperature measurement via a $\mathrm{Cu}$ - or Al- element as described above is not only used at the inner ring but also at the outer ring. However, for verifying purposes the fact that the outer ring is standing still allows for an additional temperature measurement directly on the outer ring surface.

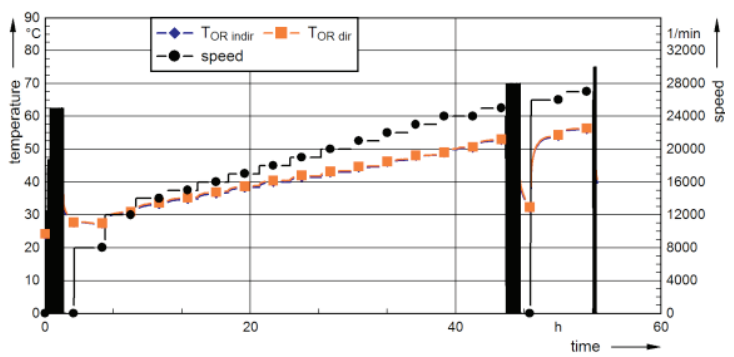

Fig. 9. Reliability of the inderect temperature measurement

Fig. 9 shows the differences in the measured outer ring temperature between the direct and indirect measurement up to $28,000 \mathrm{rpm}$. The negligible difference between the two signals proves that the indirect measurement does not affect the quality of the measurements. This makes the mechanical integration of the telemetry rotor generally suitable for the given application.

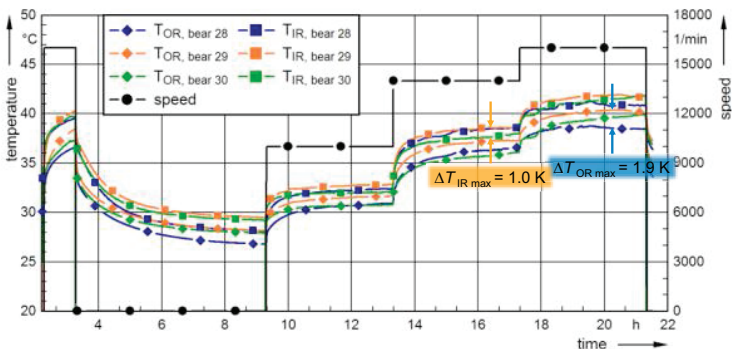

Fig. 10. Repeatability of the measurements

For three different bearings ("bear 28", "bear 29 ", "bear 30") of the same make and type that were tested on the same test rig, Fig. 10 shows the differences between the measured inner and outer ring temperatures. Differences in absolute values between the bearings result from minor variations in production quality and the process of mounting the bearing on the test rig. The fact that the maximum difference between the inner ring temperatures is even smaller than that of the outer ring temperature proves the good repeatability of the measurements.

Finally, the high speed capability of the system could be proven with test runs like that shown in Fig. 11. There the inner and outer ring temperature as well as the inner ring excess temperature of a $70 \mathrm{~mm}$ bore grease lubricated hybrid spindle bearing up to $28,000 \mathrm{rpm}$ are shown. The telemetry system is giving a signal of constantly good quality allowing for an in depth investigation of the bearing's operating 
behavior that would not be possible with a mere measurement of the outer ring temperature.

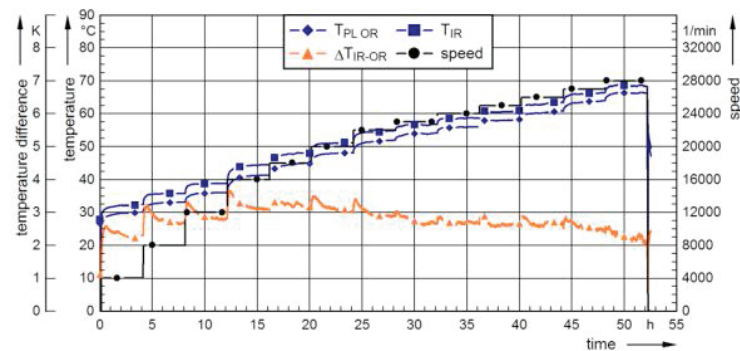

Fig. 11. Speed-temeprature behavior of a spindle bearing up to $28,000 \mathrm{rpm}$

\section{Summary}

The ever growing demand for increased productivity of machine tools and machining centers requires main spindles and thus spindle bearings with very high speed ratings and a high reliability. At speed coefficients up to $n \times d_{m}=2,7 \cdot 10^{6} \mathrm{~mm} / \mathrm{min}$ the friction induced heat inside the bearing is one of the main factors limiting the speed rating. The common case of a shaft and thus bearing inner ring excess temperature is most crucial for spindle bearings with small contact angles and especially cylindrical roller bearings. Here an excessive thermally induced growth of the inner ring can cause jamming of the bearing what causes the failure of the bearing and spindle and machine downtimes.

For the development and investigation of such bearings special custom made test benches are necessary. At WZL several such test rigs are operated for various purposes. Most of them are equipped with a telemetry system that allows for the measurement of the inner ring temperature via a Pt1000-sensor that is integrated in the shaft. In various tests the quality of the measurements as well as the high speed capability of the system could be proven.

\section{Outlook}

Especially in today's HSC applications main spindles and their bearings are often pushed to their limits. A telemetry system like the one described here can beneficially be used for continuously monitoring the spindle state. Bearing temperature and drawbar force are figures of vital importance that can help to determine the spindle state and predict necessary services before an actual spindle failure occurs. Since the high speed capability of the system has successfully been proven and especially the rotor design is very adaptable, the next level of application for this system could be spindles in an industrial manufacturing environment.

\section{References}

[1] Schaeffler Technologies: Hochgenauigkeitslager. SP1. Schweinfurt, 2011. Corporate publication

[2] Butz, F.: Gestaltung der Loslagerung von Werkzeugmaschinen. Dissertation. RWTH Aachen University. 2007

[3] DIN 51819-1: Testing of lubricants Mechanical-dynamic testing in roller bearing apparatus FE8 - Part 1: General working principles. December 1999. Standard

[4] DIN 51821-1: Testing of lubricants - Test using the $F A G$ roller bearing grease testing apparatus FE9 - General working principles. October 1988. Standard

[5] Rossaint, J.: Steigerung der Leistungsfähigkeit von Spindellagern durch optimierte Lagergeometrien. Dissertation. RWTH Aachen University. 2013

[6] Gatzen, M. M.; Poll, G.: Einfluss von polymeren Zusätzen auf die Gebrauchsdauer von Schmierfetten. Hamburg: DGMK, 2009

[7] Brecher, C.; Mayer, J.; Bäumler, S.; Reichelt, M.; Hassis, A.: Untersuchung tribologisch induzierter Randschichten in schnell drehenden Hybridwälzlagern. $53^{\text {rd }}$ Tribologie-Fachtagung. Göttingen: September $24^{\text {th }}-26^{\text {th }}, 2012$

[8] Brecher, C.; Bäumler, S.; Hassis, A.: Das Betriebsverhalten unterschiedlich formulierter Modellfette in schnell drehenden Hybridwälzlagern. $54^{\text {th }}$ Tribologie-Fachtagung. Göttingen: September $30^{\text {th }}$ - October $02^{\text {nd }}, 2013$

[9] DIN 51821-2: Testing of lubricants - Test using the FAG roller bearing grease testing apparatus FE9 - Test method A/1500/6000. March 1989. Standard

[10] Brecher, C.; Rossaint, J.; Bonerz,S.: Drahtlose Messung im Hochdrehzahlbereich. In: Konstruktion. $64^{\text {th }}$ ed., 2011 , No. $11-12$, p. 2-4

\section{Acknowledgements}

The IGF project no. $17093 \mathrm{~N}$, coordinated by the German Society for Petroleum and Coal Science and Technology (DGMK e.V.), is funded by the Federal Ministry for Economic Affairs and Energy (BMWi) on the basis of a decision by the German Bundestag as part of the AiF Industrial Collective Research (IGF) program. 